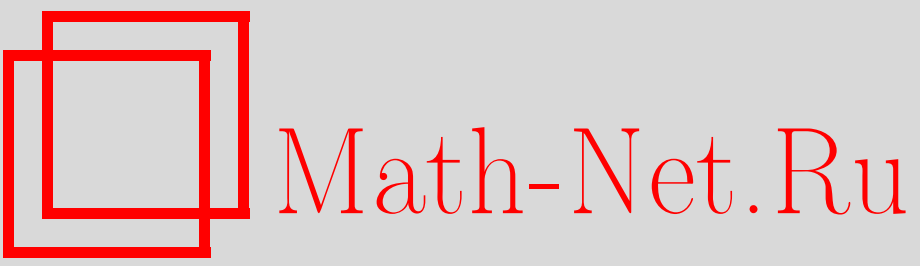

Е. Д. Трифонов, Нелинейное нестационарное уравнение Шредингера для задачи о взаимодействии бозе-эйнштейновского конденсата разреженных газов с электромагнитным полем, ТМФ, 2004, том 139, номер 3, 449-461

DOI: https://doi.org/10.4213/tmf72

Использование Общероссийского математического портала Math-Net.Ru подразумевает, что вы прочитали и согласны с пользовательским соглашением

http://www.mathnet.ru/rus/agreement

Параметры загрузки:

IP : 54.197 .217 .227

26 апреля 2023 г., 14:09:57 


\title{
НЕЛИНЕЙНОЕ НЕСТАЦИОНАРНОЕ УРАВНЕНИЕ ШРЕДИНГЕРА ДЛЯ ЗАДАЧИ О ВЗАИМОДЕЙСТВИИ БОЗЕ-ЭЙНШТЕЙНОВСКОГО КОНДЕНСАТА РАЗРЕЖЕННЫХ ГАЗОВ С ЭЛЕКТРОМАГНИТНЫМ ПОЛЕМ
}

\begin{abstract}
Приводится вывод уравнения Шредингера, описывающего взаимодействие бозеэйнштейновского конденсата идеального газа с электромагнитным полем. Его решения позволяют найти эволюцию интенсивности излучения и заселенностей когерентных атомных состояний с различными значениями импульсов отдачи и могут быть использованы для описания таких эффектов, как рассеяние и усиление света, усиление атомного пучка (атомный лазер), индуцированная прозрачность.
\end{abstract}

Ключевые слова: бозе-эйнштейновский конденсат, сверхизлучательное рассеяние, когерентное усиление, сокращение скорости света, атомный лазер.

\section{1. ВВЕДЕНИЕ}

В 1995 году впервые с помошью лазерного и испарительного охлаждений был получен бозе-эйнштейновский конденсат (БЭК) разреженных паров шелочных металлов [1]. Обстоятельные обзоры теоретических и экспериментальных достижений в этой области можно найти в работах [2]. Обший подход к проблеме взаимодействия света с вырожденным бозе-газом атомов содержится в работах [3], [4]. В частности, квантово-электродинамическое описание рассеяния света на БЭК было выполнено в статье [5]. Полуклассическая теория сверхизлучательного рассеяния для модели "одетого" атома была развита в работах [6]. Авторы этих работ эффективно исключают из рассмотрения возбужденные электронные состояния и ограничиваются базисом, состояшим из основных электронных состояний с различными импульсами поступательного движения. Записанные в таком базисе уравнения Максвелла-Блоха дополнены феноменологическими релаксационными членами, в частности членом, описьваюшим линейные потери поля

\footnotetext{
${ }^{*}$ Российский государственный педагогический университет им. А. И. Герцена, Санкт-Петербург, Россия. E-mail: thphys@herzen.spb.ru
} 
за счет выхода излучения из системы. В наших работах [7], обобщение которых представляет данная публикация, мы также используем полуклассический подход, но сохраняем в базисе возбужденные электронные состояния и явным образом решаем задачу о распространении света через систему. Так же как в упомянутых выше работах [4], мы рассматриваем БЭК как идеальный газ, пренебрегая прямыми межатомными взаимодействиями и учитывая лишь взаимодействие атомов с поперечным электромагнитным полем. Такая возможность оправдывается оценкой величины химического потенциала при температурах ниже критической. Для системы, использованной в экспериментах [8]-[11], химический потенциал на один-два порядка меньше кинетической энергии атомов, получаемой ими в процессе рассеяния.

\section{2. ЭКСПЕРИМЕНТАЛЬНЫЕ ИССЛЕДОВАНИЯ КОГЕРЕНТНЫХ ОПТИЧЕСКИХ ЭФФЕКТОВ В БЭК}

2.1. Кооперативное или сверхизлучательное рассеяние света. При обычном (спонтанном) рэлеевском рассеянии интенсивность рассеяного света линейно зависит от интенсивности падающего излучения. Падающее поле наводит в атомах дипольные моменты, которые, в свою очередь, создают вторичное (рассеянное) поле излучения. Другими словами, рассеянное поле представляет собой сумму вторичных полей, излученных отдельными атомами. Если рассеивающая среда была бы абсолютно однородной, то из-за интерференции вторичных полей возникало бы излучение только вперед - это так называемая когерентная часть рассеяния. Атом, участвуюший в когерентном рассеянии, не изменяет своего импульса и остается в состоянии бозе-эйнштейновской конденсации. При наличии флуктуаций плотности рассеиваюшей среды возникает некогерентное рэлеевское рассеяние. Интенсивность некогерентного рэлеевского рассеяния складьвается из интенсивностей рассеяния отдельными атомами. Таким образом, описание некогерентного рассеяния системой атомов сводится к задаче о рассеянии одним атомом. В случае бозе-эйнштейновской конденсации некогерентное рассеяние обусловлено квантовыми флуктуациями плотности распределения частиц.

В отличие от обычного рэлеевского рассеяния, при кооперативном рассеянии проявляется действие вторичных полей одних атомов на другие. Этот процесс родственный вынужденному рассеянию, при котором роль "вынуждаюшего" поля играет само поле рассеяния. Если кооперативное рассеяние происходит в условиях сохранения фазовой памяти атомов (что типично для БЭК), то его естественно характеризовать как сверхизлучательное.

В эксперименте [8] БЭК разреженных паров натрия был приготовлен в магнитной ловушке с помошью лазерного охлаждения и испарения. Магнитная ловушка имела вытянутую форму с размерами 20 мкм в диаметре и 200 мкм в длину. Число атомов в ловушке порядка $10^{7}$. Образец подвергался облучению лазерным импульсом, частота которого была сдвинута в красную сторону на 1.7 Гц от частоты перехода $3 S_{1 / 2} \rightarrow 3 P_{3 / 2}$. Лазерный пучок пересекал конденсат перпендикулярно его оси. Исследовались не только интенсивность рассеянного излучения, но также и распределение импульсов поступательного движения атомов, которые получались в результате взаимодействия с лазер- 


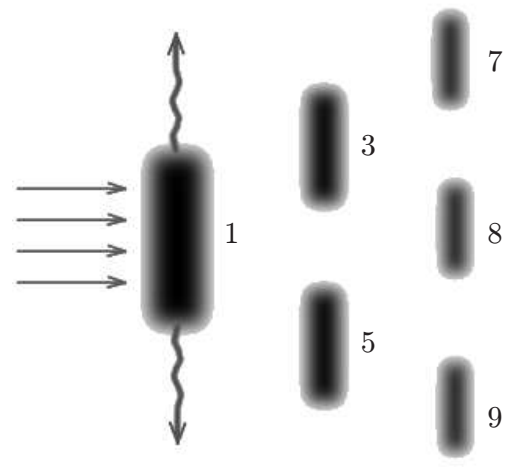

a

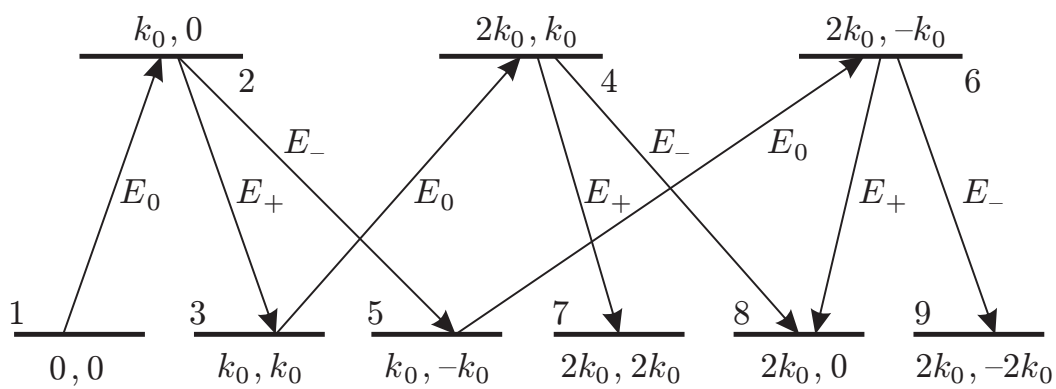

б

Рис. 1. а - Схема формирования когерентных атомных состояний с отличными от нуля импульсами в результате сверхизлучательного рассеяния света. Подлинную фотографию этих когерентных атомных "облаков" можно найти в работе [11]. б - Схема переходов между когерентными атомными состояниями в процессе сверхизлучательного рассеяния. Нумерация состояний проведена в соответствии с (16). Для каждого состояния указаны $x$ - и $y$-составляющие импульса поступательного движения атома.

ными и рассеянными фотонами. С этой целью после лазерного облучения магнитная ловушка выключалась и атомы двигались свободно по инерции. В результате облако конденсата расшеплялось на несколько частей, которые спустя некоторое время занимали в пространстве разные положения в соответствии с приобретенными скоростями (см. рис. 1a). Авторы отмечают различный эффект в зависимости от поляризации лазерного луча. При поляризации, параллельной оси образца, наблюдалось движение атомов, соответствуюшее импульсам отдачи, как при обычном (некогерентном) рэлеевском рассеянии. Однако если поляризация лазерного луча была перпендикулярна оси образца, то наблюдалось интенсивное рассеяние вдоль образца. Атом, участвующий в процессе рассеяния, получает импульс лазерного фотона, направленный вдоль лазерного луча, а при испускании рассеянного фотона - такой же импульс по величине, но направленный вдоль образца. Результирующий импульс атома оказывается направленным под углом $45^{\circ}$ к оси образца. Некоторые атомы успевают принять участие в последуюших актах рассеяния и соответственно получают дополнительные импульсы. 
2.2. Усиление света в БЭК. В экспериментах [9] по усилению света конденсат паров натрия (такой же, как в эксперименте [8]) облучался двумя лазерными пучками: одеваюшим и пробным. Оба пучка лежали в плоскости, перпендикулярной оси вытянутости конденсата, и пересекали друг друга под углом $135^{\circ}$. Основной результат этого эксперимента заключался в обнаружении усиления пробного луча при включенном одевающем луче. При низкой интенсивности пробного луча после выключения одевающего луча отчетливо наблюдалось прекращение усиления. Однако при больших интенсивностях одеваюшего луча наблюдалось остаточное усиление даже при выключении пробного луча на входе. Это своеобразный голографический эффект: пробный луч возникал на выходе благодаря дифракции одеваюшего луча на дифракционной решетке атомов, получивших импульсы отдачи. Кроме того, наблюдалось запаздывание пробного импульса на выходе, что интерпретировалось как уменьшение групповой скорости света до величины $1 \mathrm{~m} / \mathrm{c}$.

2.3. Усиление атомного пучка (атомный лазер). Схема опыта [10] по усилению атомного пучка такая же, как в экспериментах по рассеянию. Сначала с помощью аксиального и радиального (перпендикулярного к оси конденсата) лазерных импульсов в конденсате создается небольшая $\left(10^{-2}-10^{-4}\right)$ доля атомов, получивших импульс отдачи в результате вынужденного рассеяния. Как и раньше, частоты обоих лазерных пучков сдвинуты в красную сторону, при этом частота аксиального пучка несколько меньше частоты радиального на величину, соответствуюшую кинетической энергии (энергии отдачи) атома. Затем в течение некоторого времени проводится облучение только радиальным пучком более высокой мощности. Теперь уже происходит спонтанное рассеяние. Оказывается, что с большей вероятностью происходит рассеяние в аксиальном направлении в конечное состояние атома, которое было создано вначале. В результате происходит усиление интенсивности атомного пучка.

2.4. Индуцированная прозрачность и сокращение групповой скорости света. В эксперименте [11] наблюдалось сокрашение групповой скорости лазерного импульса благодаря наведению индуцированной прозрачности. Эксперимент проводился с парами натрия, охлажденными до температуры фазового перехода $T_{\mathrm{c}}=435 \mathrm{HK}$ при пиковой плотности облака $5 \cdot 10^{12} \mathrm{~cm}^{-3}$. На систему направлялись два взаимно перпендикулярных пучка: пробный и связываюший, настроенные в резонанс с переходами $1 \rightarrow 3$ и $2 \rightarrow 3$, соответственно, где состояния 1 и 2 отвечают подуровням сверхтонкой структуры основного терма $3 S_{1 / 2}$, а состояние 3 - одному из подуровней сверхтонкой структуры возбужденного состояния $3 P_{3 / 2}$. Первоначально заселенным является только уровень 1 . Более интенсивный связывающий луч создает когерентную суперпозицию состояний 2 и 3 , и в результате для перехода $1 \rightarrow 3$ возникает прозрачность в некотором узком частотном интервале. В этом же интервале возникает линейная дисперсия, приводяшая к сокрашению групповой скорости света.

\section{3. НЕЛИНЕЙНОЕ УРАВНЕНИЕ ШРЕДИНГЕРА}

Считая падающие лазерные пучки квазирезонансными с одним из электронных переходов в атоме, мы будем рассматривать каждый атом как двухуровневую или трех- 
уровневую (для задачи об индуцированной прозрачности) электронную систему. Атом будем характеризовать не только его электронным состоянием, но и состоянием поступательного движения с определенным значением импульса. Мы предполагаем, что исходно все атомы находятся в основном электронном состоянии с почти нулевым значением импульса. При взаимодействии атома с излучением выполняется закон сохранения импульса. Формально это обеспечивается правилами отбора. Матричный элемент взаимодействия атома с электромагнитной волной с волновым вектором $\mathbf{k}$ отличен от нуля для конечного состояния атома, импульс которого отличается от исходного на величину $\hbar \mathbf{k}$. Электронные состояния атома (основное и возбужденное) будем отмечать индексами $a$ и $b: \varphi_{a}, \varphi_{b}$. Поступательное движение будем описывать собственными функциями импульса. Например, базис волновых функций двухуровневого атома мы выбираем в виде

$$
\begin{aligned}
|a ; \mathbf{k}\rangle & =\frac{1}{\sqrt{V}} e^{i \mathbf{k} \cdot \mathbf{r}} \varphi_{a} \\
\left|b ; \mathbf{k}^{\prime}\right\rangle & =\frac{1}{\sqrt{V}} e^{i \mathbf{k}^{\prime} \cdot \mathbf{r}} \varphi_{b} e^{-i \omega_{0} t}
\end{aligned}
$$

где $\omega_{0}$ - частота лазерного поля, $V$ - объем системы. Отметим, что функции (1) отличаются от волновых функций свободного атома зависяшим от времени фазовым множителем, в котором вместо частоты, соответствующей собственному значению энергии атома (в которой должна быть учтена также и кинетическая энергия поступательного движения), мы берем частоту квазирезонансного лазерного поля. В общем случае временны́е фазовые множители будем выбирать так, чтобы для состояний, связанных полем, они отличались на частоту этого поля. Выбранное на таком базисе представление отличается от обычно используемого представления "взаимодействия", и соответствующие поправки появятся в уравнении Шредингера. Общее выражение для волновой функции атома может быть записано в виде

$$
\Psi=\sum_{a, \mathbf{k}} C_{a, \mathbf{k}}|a ; \mathbf{k}\rangle
$$

где $C_{a, \mathbf{k}}-$ зависящие от времени коэффищиенты разложения. Состояния всех атомов мы предполагаем тождественными в соответствии с требованием симметрии волновой функции системы бозе-частиц. Элементы соответствуюшей матрицы плотности могут быть представлены в виде

$$
R_{a, \mathbf{k} ; b, \mathbf{k}^{\prime}}=C_{a, \mathbf{k}} \overline{C_{b, \mathbf{k}^{\prime}}}
$$

где чертой обозначено комплексное сопряжение.

Мы будем рассматривать взаимодействие атомов, образующих БЭК, с $n$ модами электромагнитного поля $\mathcal{E}_{j}(y, t)$, имеющими форму плоских волн с хорошо различимыми волновыми векторами $\mathbf{k}_{j}$. Очевидно, что взаимодействие с модой $\mathcal{E}_{j}$ связывает состояния $|a, \mathbf{k}\rangle$ и $\left|b, \mathbf{k}^{\prime}\right\rangle$, только если $\mathbf{k}^{\prime}-\mathbf{k}= \pm \mathbf{k}_{j}$. Используя оператор взаимодействия атома с электромагнитным полем в виде $\widehat{H}_{\mathrm{int}}=-\hat{d} \mathcal{E}$, где $\hat{d}$ - оператор электронного 
дипольного момента атома, запишем уравнение Шредингера для одного атома. В приближении вращающейся волны имеем

$$
\begin{aligned}
i \hbar \dot{C}_{a, \mathbf{k}} & =\sum_{j}\left\langle a, \mathbf{k}\left|\hat{d} \mathcal{E}_{j}\right| b, \mathbf{k}+\mathbf{k}_{j}\right\rangle C_{b, \mathbf{k}+\mathbf{k}_{j}}-i \varepsilon_{k} C_{a, \mathbf{k}}, \\
i \hbar \dot{C}_{b, \mathbf{k}} & =\sum_{j}\left\langle b, \mathbf{k}\left|\hat{d} \mathcal{E}_{j}\right| a, \mathbf{k}-\mathbf{k}_{j}\right\rangle C_{a, \mathbf{k}-\mathbf{k}_{j}}-\left(\gamma+i \varepsilon_{k}+i \Delta\right) C_{b, \mathbf{k}},
\end{aligned}
$$

где $\Delta=\omega_{b a}-\omega_{0}-$ расстройка, $\omega_{a b}$ - частота перехода между состояниями $b$ и $a, \varepsilon_{k}-$ кинетическая энергия атома, обладаюшего импульсом $\mathbf{k}, \gamma$ - радиационная константа возбужденного электронного состояния атома, которая описывает спонтанное рассеяние света в произвольных направлениях.

Каждая из мод создает поляризованность среды (конденсата), и, в свою очередь, поляризованность индуцирует вторичное поле этой же моды $\mathcal{E}_{j}^{\prime}$. В одномерном случае вторичное поле выражается через поляризованность следующим образом:

$$
\mathcal{E}_{j}^{\prime}(y, t)=\frac{2 \pi i \omega_{j}}{c} \int_{0}^{L} P_{j}\left(y^{\prime}, t-\frac{\left|y-y^{\prime}\right|}{c}\right) e^{i k_{j}\left|y-y^{\prime}\right|} e^{-i \omega_{j} t} d y^{\prime}+\text { к.c. }
$$

где $c$ - скорость света в вакууме, $P_{j}\left(y^{\prime}, t\right)$ - комплексные амплитуды поляризованности среды, $y$ - координата рассматриваемой моды, $L$ - длина конденсата вдоль этой координаты. Эти амплитуды поляризованности, в свою очередь, выражаются через недиагональные элементы матрицы плотности как среднее квантово-механическое значение оператора поляризованности

$$
\widehat{P}_{j}=\sum_{\alpha} \hat{d}_{\alpha} \delta\left(\mathbf{r}-\mathbf{r}_{\alpha}\right)
$$

где индекс $\alpha$ нумерует атомы конденсата, $\mathbf{r}_{\alpha}$ - радиус-вектор центра инерции $\alpha$-го атома, $\hat{d}_{\alpha}$ - оператор дипольного момента $\alpha$-го атома. Используя базисные функции (1), получаем

$$
P_{j}\left(y^{\prime}, t\right)=N_{0} d e^{i k_{j} y^{\prime}} \sum_{\mathbf{k}-\mathbf{k}^{\prime}=\mathbf{k}_{j}} R_{b, \mathbf{k} ; a, \mathbf{k}^{\prime}}
$$

где $N_{0}$ - концентрация атомов в конденсате, $d$ - матричный элемент дипольного момента перехода. Отметим, что зависимость поляризованности $P_{j}$ от поля $\mathcal{E}_{j}$ оказывается нелокальной. Поле входит лишь в матричные элементы взаимодействия (в уравнении Шредингера) и интегрируется по всему объему конденсата. Поэтому амплитуды $C_{a, \mathbf{k}}$, $C_{b, \mathbf{k}}$ разложения волновой функции, являющиеся решением уравнения Шредингера (4), а следовательно, и элементы матрицы плотности $R_{b, \mathbf{k} ; a, \mathbf{k}^{\prime}}$ и поляризованность $(7)$ зависят от поля нелокально.

Пренебрегая запаздыванием в амплитуде поляризованности и вкладом быстроосциллируюших членов при интегрировании по $y^{\prime}$ в $(5)$, получаем

$$
\mathcal{E}_{j}^{\prime}(y, t)=\frac{2 \pi}{c} i \omega_{j} d N_{0} y e^{-i \omega_{j} t} e^{i k_{j} y} \sum_{\mathbf{k}^{\prime}-\mathbf{k}=\mathbf{k}_{j}} R_{b, \mathbf{k} ; a, \mathbf{k}^{\prime}}
$$


Как видно, поле, индуцированное пространственно однородной волной поляризации, линейно по $y$. Это обусловлено нелокальной зависимостью поляризованности от поля: поляризованность в данной точке конденсата не определяется полем в этой точке, а зависит интегрально от поля во всем объеме конденсата. Далее в выражении для матричных элементов в уравнении Шредингера (4) мы заменим эту величину средним значением, положив $y=L / 2$. Кроме того, выберем величину $\hbar / \tau_{R} d$ в качестве единицы напряженности электрического поля, где

$$
\tau_{R}=\frac{c \hbar}{\pi \omega_{0} d^{2} N_{0} L}
$$

Саму величину $\tau_{R}$ мы выберем в качестве единицы времени. Напомним, что квадрат модуля амплитуды поля в выбранных единицах равен числу фотонов, испускаемых одним атомом за время $\tau_{R}$ (см., например, [12]). Подставляя выражение для поля (8) в уравнение (4), получим

$$
\begin{aligned}
\dot{C}_{a, \mathbf{k}} & =\sum_{j} \overline{E_{j}} C_{b, \mathbf{k}+\mathbf{k}_{j}}-i \varepsilon_{k} C_{a, \mathbf{k}}, \\
\dot{C}_{b, \mathbf{k}} & =-\sum_{j} E_{j} C_{a, \mathbf{k}-\mathbf{k}_{j}}-\left(\gamma+i \varepsilon_{k}+i \Delta\right) C_{b, \mathbf{k}},
\end{aligned}
$$

где

$$
E_{j}=E_{j}^{0}+\sum_{\mathbf{k}^{\prime}-\mathbf{k}^{\prime \prime}=\mathbf{k}_{j}} C_{b, \mathbf{k}^{\prime}} \overline{C_{a, \mathbf{k}^{\prime \prime}}}
$$

Здесь величины $E_{j}$ определяются с точностью до фазового множителя - частоты Раби соответствуюших полей в единицах $\tau_{R}^{-1}, E_{j}^{0}$ - частота Раби падаюшего (или флуктуационного) поля. Все эти параметры также записаны в единицах $\tau_{R}$. Поскольку амплитуды полей выражаются через коэффициенты разложения волновой функции по выбранному базису состояний, то уравнение Шредингера оказывается нелинейным. Напомним, что во всех внешних полях мы выделили зависимость от времени на некоторой основной частоте $\omega_{0}$. Поэтому если какие-то внешние поля имеют другую несушую частоту, то в амплитуду этой моды должен быть добавлен соответствуюший временной фазовый множитель.

Для учета релаксации брэгговской решетки, образованной когерентными состояниями атомов с определенными значениями импульсов, от уравнения Шредингера (10) сле- 
дует перейти к уравнению для матрицы плотности:

$$
\begin{aligned}
\dot{R}_{a, \mathbf{k} ; a, \mathbf{k}^{\prime}}= & -\Gamma_{\mathbf{k}, \mathbf{k}^{\prime}} R_{a, \mathbf{k} ; a, \mathbf{k}^{\prime}}+\sum_{j}\left\{\overline{E_{j}} R_{b, \mathbf{k}+\mathbf{k}_{j} ; a, \mathbf{k}^{\prime}}+E_{j} R_{a, \mathbf{k} ; b, \mathbf{k}^{\prime}+\mathbf{k}_{j}}\right\}+ \\
& +i\left(\varepsilon_{k^{\prime}}-\varepsilon_{k}\right) R_{a, \mathbf{k} ; a, \mathbf{k}^{\prime}} \\
\dot{R}_{a, \mathbf{k} ; b, \mathbf{k}^{\prime}}= & \sum_{j}\left\{\overline{E_{j}} R_{b, \mathbf{k}+\mathbf{k}_{j} ; b, \mathbf{k}^{\prime}}-E_{j} R_{a, \mathbf{k} ; a, \mathbf{k}^{\prime}-\mathbf{k}_{j}}\right\}+ \\
& +i\left(\varepsilon_{k^{\prime}}-\varepsilon_{k}-\frac{\gamma_{b}}{2}+i \Delta\right) R_{a, \mathbf{k} ; b, \mathbf{k}^{\prime}} \\
\dot{R}_{b, \mathbf{k} ; b, \mathbf{k}^{\prime}}= & -\sum_{j}\left\{E_{j} R_{a, \mathbf{k}-\mathbf{k}_{j} ; b, \mathbf{k}^{\prime}}+\overline{E_{j}} R_{b, \mathbf{k} ; a, \mathbf{k}^{\prime}-\mathbf{k}_{j}}\right\}+ \\
& +i\left(\varepsilon_{k^{\prime}}-\varepsilon_{k}-\gamma_{b}\right) R_{b, \mathbf{k} ; b, \mathbf{k}^{\prime}}
\end{aligned}
$$

где $\Gamma_{\mathbf{k}, \mathbf{k}^{\prime}}$ - константы поперечной релаксации.

3.1. Сверхизлучательное рассеяние. В соответствии с условиями эксперимента [11] (см. п. 2.1) мы будем рассматривать взаимодействие атомов с тремя плоскими волнами электромагнитного поля: возбуждаюший лазерный луч будем считать направленным перпендикулярно оси конденсата, а рассеянные волны - вдоль конденсата в противоположных направлениях. Обозначим эти поля соответственно через $E_{0}, E_{+}, E_{-}$, а их волновые векторы - соответственно $\mathbf{k}_{0}, \mathbf{k}_{+}, \mathbf{k}_{-}$.

Кроме процессов взаимодействия с полями $E_{0}, E_{+}, E_{-}$имеет место некогерентное рассеяние в произвольном направлении. Некогерентное (спонтанное) рассеяние является не только альтернативным процессом, но и инициирующим фактором для кооперативного рассеяния. Оценим интенсивность $W$ (число фотонов за время $\tau_{R}$ ) спонтанного рассеяния (одним атомом) в телесном угле образца. Энергия излучения диполя $d$, осциллируюшего с частотой $\omega_{0}$, в направлении, перпендикулярном диполю, в телесный угол $d \Omega$ равна

$$
d I=\frac{d^{2} \omega_{0}^{4}}{4 \pi c^{3}} d \Omega .
$$

Представляя средний дипольный момент атома, наведенный лазерным полем, как $d \cdot R_{a, 0 ; b, \mathbf{k}_{0}}$, мы получим следуюшее выражение для интересуюшей нас интенсивности:

$$
W=\frac{\left|R_{a, 0 ; b, \mathbf{k}_{0}}\right|^{2}}{\lambda^{2} N_{0} L} \frac{D^{2}}{L^{2}},
$$

где $\lambda=2 \pi c / \omega_{0}, D$ - поперечный размер конденсата. Отсюда следует, что амплитуда флуктуационного поля (в наших единицах) равна $E_{\mathrm{fl}}=\left|R_{a, 0 ; b, \mathbf{k}_{0}}\right| \cdot s$, где

$$
s=\left(\frac{1}{N_{0} \lambda^{2} L}\right)^{1 / 2} \frac{D}{L} .
$$

В записи уравнения Шредингера мы добавим это поле к полям $E_{ \pm}$как затравочное. Для условий эксперимента [11] параметр $s$ имеет порядок 0.001 . 
Условимся направление лазерного луча принимать за ось $X$, а направление вытянутости конденсата - за ось $Y$. Тогда система состояний атома, необходимая для описания рассеяния, будет иметь вид $\left|a ; k_{x}, k_{y}\right\rangle,\left|b ; k_{x}^{\prime}, k_{y}^{\prime}\right\rangle$.

Ограничиваясь актами не более чем двукратного рассеяния, пронумеруем эти состояния одним индексом следуюшим образом:

$$
\begin{array}{lll}
\Psi_{1}=|a ; 0,0\rangle, & \Psi_{2}=\left|b ; k_{0}, 0\right\rangle, & \Psi_{3}=\left|a ; k_{0},-k_{0}\right\rangle, \\
\Psi_{4}=\left|b ; 2 k_{0},-k_{0}\right\rangle, & \Psi_{5}=\left|a ; k_{0}, k_{0}\right\rangle, & \Psi_{6}=\left|b ; 2 k_{0}, k_{0}\right\rangle, \\
\Psi_{7}=\left|a ; 2 k_{0},-2 k_{0}\right\rangle, & \Psi_{8}=\left|a ; 2 k_{0}, 0\right\rangle, & \Psi_{9}=\left|a ; 2 k_{0}, 2 k_{0}\right\rangle .
\end{array}
$$

Переходы между этими состояниями поясняются диаграммой на рис. 1б. Отметим, что собственные частоты этих переходов отличаются друг от друга из-за вклада в каждый уровень кинетической энергии атома.

В соответствии с (10) по схеме переходов можно написать уравнение Шредингера. Для этого удобно придерживаться следуюших правил:

Каждому уровню на схеме сопоставляется амплитуда состояния - коэффициент разложения волновой функции по выбранному базису, каждому вектору перехода - напряженность одной из мод әлектромагнитного поля.

Уравнение для производной по времени амплитудь возбужденного состояния в правой части содержит столько слагаемых, сколько имеется связанных с ним переходами основных әлектронных состояний. Каждое из этих слагаемых имеет вид произведения напряженности указанной моды поля на амплитуду соответствующего основного әлектронного состояния со знаком минус. Кроме того, имеются слагаемье, учитьвающие кинетическую энергию рассматриваемого возбужденного состояния, отстройку от резонанса (для әлектронного перехода) и константу радиационного затухания.

Уравнение для производной по времени основного әлектронного состояния в правой части содержит столько слагаемых, сколько имеется связанных с ним переходами возбужденных әлектронных состояний. Каждое из әтих слагаемых имеет вид произведения комплексно-сопряжсенного значения напряженности указанной моды на амплитуду соответствующего возбужденного состояния. Кроме того, имеется слагаемое, учитывающее кинетическую әнергию рассматриваемого основного состояния.

Амплитуды напряженностей полевых мод представляют собой сумму амплитуд внешних (или флуктуационных) полей и амплитуды вторичного поля. Амплитуда вторичного поля представляет собой сумму произведений комплексно-сопряженной амплитуды основного состояния на амплитуду возбужденного состояния, которые связаны рассматриваемой модой поля.

В нашем случае (для схемы переходов, изображенной на рис. 1б) уравнение Шредин- 
гера (10) будет иметь вид

$$
\begin{aligned}
& \dot{C}_{1}=\overline{E_{0}} C_{2}, \\
& \dot{C}_{2}=\left(i \Delta-\frac{\gamma}{2}\right) C_{2}-E_{+} C_{3}-E_{-} C_{5}-E_{0} C_{1}, \\
& \dot{C}_{3}=-i \varepsilon_{3} C_{3}+\overline{E_{+}} C_{2}+\overline{E_{0}} C_{4}, \\
& \dot{C}_{4}=\left(i \Delta-\frac{\gamma}{2}\right) C_{4}-E_{+} C_{7}-E_{-} C_{8}-E_{0} C_{3}, \\
& \dot{C}_{5}=-i \varepsilon_{5} C_{5}+\overline{E_{-}} C_{2}+\overline{E_{0}} C_{6}, \\
& \dot{C}_{6}=\left(i \Delta-\frac{\gamma}{2}\right) C_{6}-E_{+} C_{8}-E_{-} C_{9}-E_{0} C_{5}, \\
& \dot{C}_{7}=-i \varepsilon_{7} C_{7}+\overline{E_{+}} C_{4}, \\
& \dot{C}_{8}=-i \varepsilon_{8} C_{8}+\overline{E_{+}} C_{6}+\overline{E_{-}} C_{4}, \\
& \dot{C}_{9}=-i \varepsilon_{9} C_{9}+\overline{E_{-}} C_{6} .
\end{aligned}
$$

Здесь $C_{i}$ - коэффициенты разложения волновой функции по выбранному ранее базиcy (16), a

$$
\begin{aligned}
& E_{+}=\overline{C_{3}} C_{2}+\overline{C_{7}} C_{4}+\overline{C_{8}} C_{6}+s\left|\overline{C_{2}} C_{1}\right|, \\
& E_{-}=\overline{C_{5}} C_{2}+\overline{C_{8}} C_{4}+\overline{C_{9}} C_{6}+s\left|\overline{C_{2}} C_{1}\right|
\end{aligned}
$$

в соответствии с (10). В уравнениях для амплитуд возбужденных состояний мы пренебрегли слагаемым с кинетической энергией атомов по сравнению с членом, учитываюшим отстройку. При описанной выше постановке задачи единственным ненулевым начальным условием будет $C_{1}(0)=1$.

Отметим, что поля рассеяния, которые будут регистрироваться на выходе, в два раза больше средних полей в образце: $E_{\text {out }}^{ \pm}=2 E_{ \pm}$(из-за отмеченной вьше линейной зависимости по пространственной координате).

3.2. Когерентное усиление света. Схема переходов, описываюшая этот эффект [9] (см. п. 2.2) при ограничении актами возбуждения первого порядка, приведена на рис. 2, где $E_{\mathrm{d}}$ и $E_{\mathrm{p}}$ - амплитуды одеваюшего и пробного полей, а $\mathbf{k}_{\mathrm{d}}$ и $\mathbf{k}_{\mathrm{p}}-$ их волновые векторы. В качестве базиса волновых функций выбираем

$$
\begin{gathered}
\Psi_{1}=|a ; 0\rangle, \quad \Psi_{2}=\left|b ; \mathbf{k}_{\mathrm{d}}\right\rangle, \quad \Psi_{3}=\left|a ; \mathbf{k}_{\mathrm{d}}-\mathbf{k}_{\mathrm{p}}\right\rangle, \\
\Psi_{4}=\left|b ; \mathbf{k}_{\mathrm{p}}\right\rangle, \quad \Psi_{5}=\left|b ; \mathbf{k}_{\mathrm{p}}-\mathbf{k}_{\mathrm{d}}\right\rangle .
\end{gathered}
$$

Уравнение Шредингера (10) в данном случае принимает вид

$$
\begin{aligned}
& \dot{C}_{1}=\overline{E_{\mathrm{d}}} C_{2}+\overline{E_{\mathrm{p}}} C_{4}, \\
& \dot{C}_{2}=-E_{\mathrm{d}} C_{1}-E_{\mathrm{p}} C_{3}-\left(\gamma+i \varepsilon_{2}+i \Delta\right) C_{2}, \\
& \dot{C}_{3}=\overline{E_{\mathrm{p}}} C_{2}-i \varepsilon_{3} C_{3}, \\
& \dot{C}_{4}=-E_{\mathrm{p}} C_{1}-E_{\mathrm{d}} C_{3}-\left(\gamma+i \varepsilon_{4}+i \Delta\right) C_{4}, \\
& \dot{C}_{5}=\overline{E_{\mathrm{d}}} C_{4}-i \varepsilon_{5} C_{5},
\end{aligned}
$$




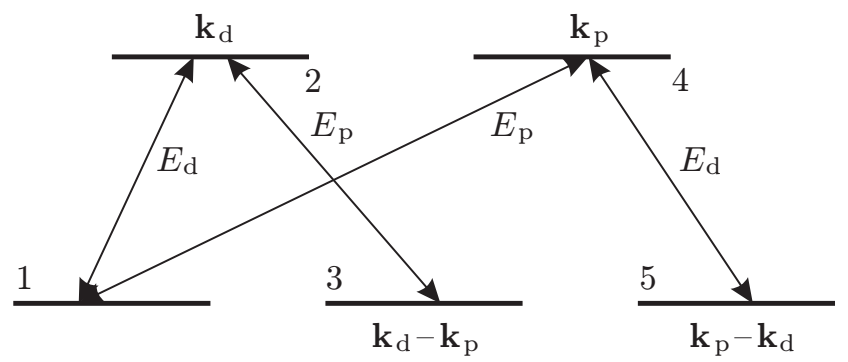

Рис. 2. Схема атомных переходов для описания усиления пробного поля $E_{\mathrm{p}}$ в присутствии одевающего поля $E_{\mathrm{d}}$.

где

$$
E_{\mathrm{d}}=E_{\mathrm{d}}^{0}+\overline{C_{1}} C_{2}+\overline{C_{5}} C_{4}, \quad E_{\mathrm{p}}=E_{\mathrm{p}}^{0}+\overline{C_{1}} C_{4}+\overline{C_{3}} C_{2}
$$

Так же как в предыдущей задаче, единственным отличным от нуля начальным условием будет $C_{1}(0)=1$, а поле на выходе равно

$$
E_{\mathrm{p}}^{\mathrm{out}}=E_{\mathrm{p}}^{0}+2\left(\overline{C_{1}} C_{4}+\overline{C_{3}} C_{2}\right)
$$

3.3. Усиление атомных пучков. В соответствии с описанием эксперимента (см. п. 2.3) постановка этой задачи аналогична формулировке задачи о рассеянии. Отличие заключается в том, что поле $E_{+}^{0}$ на первой стадии процесса должно помимо флуктуационной части иметь слагаемое, играющее роль затравочного поля. Последнее вместе с радиальным полем такой же интенсивности создает облако атомов с импульсом $\mathbf{k}_{0}-\mathbf{k}_{+}$. На второй стадии процесса затравочное поле $E_{+}^{0}$ выключается, а радиальное поле усиливается. В течение второй стадии исследуется усиление потока атомов, возникшего на первой стадии. Для оценки величины усиления интенсивность облака с импульсом $\mathbf{k}_{0}-\mathbf{k}_{+}$можно сравнить с интенсивностью облака атомов с импульсом $\mathbf{k}_{0}-\mathbf{k}_{-}$.

3.4. Сокращение групповой скорости светового импульса. В отличие от рассмотренных выше эффектов, в которых применялась двухуровневая модель атома, здесь будет использована модель трехуровневого атома. Схема переходов представлена на рис. 3. Поскольку эффект носит резонансный характер, то каждому из трех электронных уровней однозначно может быть приписано значение импульса атома. Основному состоянию 1 соответствует нулевое значение импульса. Возбужденному состоянию 3 может быть приписано значение импульса, равное импульсу фотона $\mathbf{k}_{\mathrm{p}}$ пробного луча с амплитудой $E_{\mathrm{p}}^{0}$. Переход в состояние 2 из состояния 3 происходит под действием связывающего луча с амплитудой $E_{\mathrm{c}}^{0}$ и с импульсом фотона $\mathbf{k}_{\mathrm{c}}$. Поэтому электронному состоянию 2 соответствует значение импульса атома $\mathbf{k}_{\mathrm{p}}-\mathbf{k}_{\mathrm{c}}$. Уравнение Шредингера (10) для этой двухмодовой задачи с тремя уровнями принимает вид

$$
\begin{aligned}
& \dot{C}_{1}=C_{3} \overline{E_{\mathrm{p}}}, \\
& \dot{C}_{2}=C_{3} \overline{E_{\mathrm{c}}}, \\
& \dot{C}_{3}=-C_{1} E_{\mathrm{p}}-C_{2} E_{\mathrm{c}},
\end{aligned}
$$




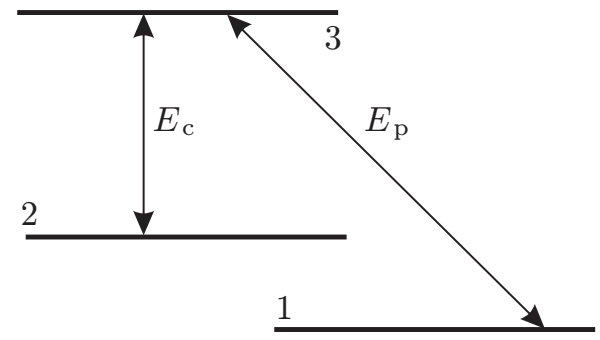

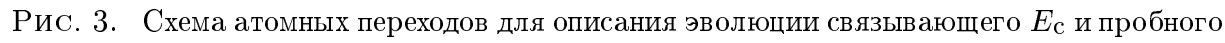
$E_{\mathrm{p}}$ полей.

где

$$
E_{\mathrm{c}}=E_{\mathrm{c}}^{0}+\overline{C_{2}} C_{3}, \quad E_{\mathrm{p}}=E_{\mathrm{p}}^{0}+\overline{C_{1}} C_{3}
$$

В соответствии с линейной зависимостью вторичного поля от длины усиления значение пробного поля на выходе полагается равным $E_{\mathrm{p}}=E_{\mathrm{p}}^{0}+2 \overline{C_{1}} C_{3}$.

\section{4. ЗАКЛЮЧЕНИЕ}

C помошью единого подхода рассмотрена теория различных явлений, которые наблюдались экспериментально: рассеяние света [8], усиление пробного луча в присутствии одевающего [9], усиление атомного пучка [10], индуцированная прозрачность, приводящая к сокрашению групповой скорости света [11].

В заключение еше раз подчеркнем роль БЭК в рассмотренных эффектах. В нашей модели БЭК представляет собой разреженный идеальный газ. В начальный момент времени атомы находятся в основном электронном состоянии с нулевым значением импульса поступательного движения. В первом приближении отсутствуют релаксационные процессы, кроме радиационных, связывающих основное и возбужденное электронные состояния атома. Благодаря этому сохраняется фазовая корреляция между различными состояниями атомов. Формально это означает, что возникают долгоживущие недиагональные элементы матришы плотности, соответствуюшие этим состояниям. Это придает сверхизлучательный характер взаимодействию атомов с излучением. Однако, в отличие от обычной теории сверхизлучения [12], [13], в которой атомы предполагаются пространственно локализованными, здесь они делокализованы по всему объему БЭК (как в состоянии с определенным значением импульса). В результате амплитуды волн поляризованности оказываются пространственно однородными и зависящими от среднего по объему значения поля. Это в определенной степени даже упрощает аппарат теории: система уравнений в частных производных сводится к обыкновенному диференциальному уравнению - уравнению Шредингера (10). В тех же случаях, когда для уточнения описания требуется учет релаксационных процессов, нарушаюших когерентность брэгговской решетки атомов, вместо уравнения Шредингера (10) следует использовать уравнение (12) для матрицы плотности.

В этой работе мы ограничились лишь формулировкой задачи, т.е. выводом нелинейного уравнения Шредингера и его записью в базисах атомных состояний, соответству- 
ющих рассматриваемым эффектам. Предварительные результаты численных решений этих уравнений (для эффектов, описанных в п. 2.1, 2.2, 3.1 и 3.2 ) приведены в наших работах [7]. Более подробный анализ полученных результатов будет приведен в последуюших публикациях.

Благодарности. Автор выражает благодарность В. Кеттерле, Л. П. Питаевскому, В. И. Юкалову за интерес к работе, М. Бенедикту и А. С. Трошину за многочисленные обсуждения затронутых в статье вопросов, Н.А. Васильеву за помошь в оформлении текста. Работа выполнена при финансовой поддержке Министерства образования РФ, гранта INTAS № 01-855 и РФФИ № 04-02-17559.

\section{Список литературы}

[1] K. B. Davis, M. -O. Mewes, M. R. Andrews, N. J. van Druten, D. S. Durfee, M. D. Kurn, W. Ketterle. Phys. Rev. Lett. 1995. V. 75. P. 3969; M. H. Anderson, J. R. Ensher, M. R. Matthews, C. E. Wieman, E. A. Cornel. Science. 1995. V. 269. P. 198.

[2] F. Dalfovo, S. Giorgini, L. P. Pitaevskii, S. Stringari. Rev. Mod. Phys. 1999. V. 71. P. 463; Ph. W. Courteille, V. S. Bagnato, V. I. Yukalov. Laser Phys. 2001. V. 11. P. 659.

[3] G. Lenz, P. Meystre, E. M. Wright. Phys. Rev. A. 1994. V. 50. P. 1681.

[4] Y. Castin, K. Mølmer. Phys. Rev. A. 1995. V. 51. P. R3426; J. Javanainen. Phys. Rev. Lett. 1995. V. 75. P. 1927

[5] M. G. Moore, P. Meystre. Phys. Rev. Lett. 1999. V. 83. P. 5202.

[6] O. E. Mustecaplioglu, L. You. Phys. Rev. A. 2000. V.62. P. 063615; N. Piovella, M. Gatelli, R. Bonifacio. Optics Commun. 2001. V. 194. P. 167.

[7] Е. Д. Трифонов. ЖЭТФ. 2001. Т. 120. С. 1117; Оптика и спектр. 2002. Т. 92. С. 631; E. D. Trifonov. Laser Phys. 2002. V. 12. P. 211.

[8] S. Inouye, A. P. Chikkatur, D. M. Stamper-Kurn, J. Stenger, D. E. Pritchard, W. Ketterle. Science. 1999. V. 285. P. 571.

[9] S. Inouye, R. F. Low, S. Gupta, T. Pfau, A. Gustavson, D. E. Pritchard, W. Ketterle. Phys. Rev. Lett. 2000. V. 85. P. 4225.

[10] S. Inouye, T. Pfau, S. Gupta, A. P. Chikkatur, A. Gorlitz, D. E. Pritchard, W. Ketterle. Lett. Nature. 1999. V. 402. P. 641.

[11] L. V. Hau, S. E. Harris, Z. Dutton, C. H. Behoroozi. Nature. 1999. V. 397. P. 594.

[12] M. G. Benedict, A. M. Ermolaev, V. A. Malyshev, I. V. Sokolov, E. D. Trifonov. Super-radiance: Multiatomic Coherent Emission. Bristol, Philadelphia: IOP, 1996.

[13] R. H. Dicke. Phys. Rev. 1954. V. 93. P. 99. 